\title{
COMPARACIÓN DE LA PRECISIÓN DE DOS LOCALIZADORES ELECTRÓNICOS APICALES EN PIEZAS DENTARIAS CON PERFORACIONES SIMULADAS
}

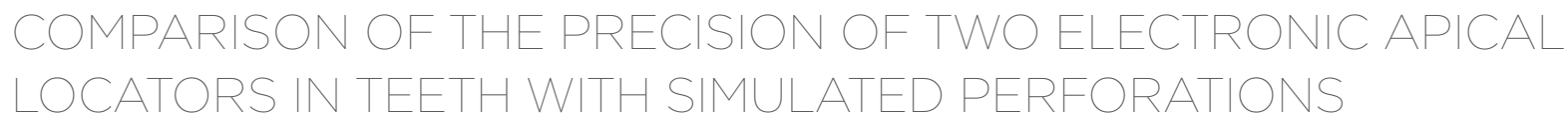

Teresa Flora Astocaza Reátegui * (i) tere.22694@gmail.com

Lourdes Rocío Ayarza Flórez ${ }^{1(1)}$ rocioayarza@hotmail.com

\section{Carmen Rosa García Rupaya' ${ }^{1(1)}$} gcarmen_rosa@hotmail.com

Artículo recibido: 08/03/2021

Arbitrado por pares

Artículo aceptado: 06/05/2021

Artículo publicado: 21/06/2021

* Autor corresponsal:

Teresa Flora Astocaza Reátegui

Email: tere.22694@gmail.com

Citar como: Astocaza T, Ayarza L, García C. Comparación de la precisión de dos localizadores electrónicos apicales en piezas dentarias con perforaciones simuladas. Rev Cient Odontol (Lima). 2021; 9 (2): e055.

DOI: $10.21142 / 2523-2754-0902-2021-055$

\section{RESUMEN}

Objetivo: El propósito de este estudio fue comparar la precisión de los localizadores electrónicos apicales Propex Pixi y Raypex 6 en la determinación de la longitud de trabajo de piezas dentarias con perforaciones simuladas a diferentes niveles del conducto radicular. Materiales y Métodos: Se utilizaron 36 premolares inferiores unirradiculares con un conducto, divididos aleatoriamente en tres grupos de 12 piezas dentarias cada uno. En el primer grupo, se realizaron dos perforaciones simuladas que fueron a nivel medio y a nivel apical; en el segundo grupo, se realizó una perforación simulada a nivel cervical, y en el tercer grupo, se realizó una perforación simulada a nivel apical. Se emplearon dos localizadores electrónicos apicales Propex Pixi y Raypex 6. La longitud real del conducto fue medida con un calibrador Vernier digital. Se aplicó estadística descriptiva y estadística inferencial con la prueba de Friedman a un nivel de confianza del 95\%. Resultados: Al comparar la precisión de las longitudes obtenidas por los localizadores electrónicos apicales Propex Pixi y Raypex 6 en premolares inferiores con perforaciones simuladas a nivel medio y apical, premolares inferiores con perforación simulada a nivel cervical y premolares inferiores con perforación simulada a nivel apical, se encontraron diferencias estadísticamente significativas en la longitud real del conducto, $\mathrm{p}<0,001, \mathrm{p}=0,008$ y $\mathrm{p}=0,006$, respectivamente. Conclusiones: El localizador electrónico apical Propex Pixi (Dentsply Maillefer, Alemania) presentó mayor precisión en la determinación de la longitud real del conducto en piezas dentarias con perforaciones simuladas a diferentes niveles del conducto radicular.

Palabras clave: endodoncia, dientes premolares, alginato, ápice del diente

\begin{abstract}
Objective: The purpose of the study was to compare the precision of the Propex Pixi and Raypex 6 electronic apical locators in determining the working length of teeth with simulated perforations at different levels of the root canal. Materials and Methods: 36 uniradicular lower premolars were used with a canal randomly divided into three groups of 12 teeth each. In the first group two simulated perforations were made at the middle and apical level. In the second group a simulated cervical perforation was made, and in the third group a simulated perforation was performed at the apical level. Two electronic apical locators Propex Pixi and Raypex 6 were used, and the real length of the canal was measured with a digital Vernier caliper. Descriptive and inferential statistics were applied with the Friedman test at a confidence level of 95\%. Results: When comparing the precision of the lengths obtained by the Propex Pixi and Raypex 6 apical electronic locators, statistically significant differences were found in the real length of the canal $(p=0.006)$ between simulated perforation of lower premolars at the cervical $(\mathrm{p}<0.001)$ and the apical level $(\mathrm{p}=0.008)$, Conclusion: The Propex Pixi apical electronic locator (Dentsply Maillefer - Germany) presented greater precision in the determination of the real canal length in teeth with simulated perforations at different levels of the root canal.
\end{abstract}

Keywords: endodontics; premolar teeth; alginates; tooth apex.

1 División de Carielogía y Endodoncia, Carrera de Estomatología, Universidad Científica del Sur. Lima, Perú. 


\section{INTRODUCCIÓN}

La intención del tratamiento endodóntico es eliminar los microorganismos de los conductos radiculares infectados mediante un procedimiento biomecánico combinado con terapia antibacteriana para lograr la cicatrización del tejido periapical. La unión cemento-dentina se considera al límite de seguridad para tener éxito en el tratamiento y conseguir el cierre biológico a expensas del cemento ${ }^{(1)}$.

Las perforaciones radiculares son complicaciones que ocurren en un $3 \%$ a un $10 \%$ de los tratamientos endodónticos, según investigaciones de Zmener y Luberti $\left({ }^{2}\right)$. Estas perforaciones pueden estar en cualquiera de los tercios radiculares y frecuentemente son producidas por iatrogenias. El uso de localizadores electrónicos apicales (LEA) es importante para su diagnóstico y localización ${ }^{3,4}$ ). Para determinar el nivel de las perforaciones radiculares, se observará si están en la raíz o en la corona del diente. De presentarse en la raíz, se localizan en el tercio cervical, tercio medio o tercio apical, y de presentarse en la corona, se localizan en el tercio cervical $\left({ }^{4,5}\right)$. Una perforación es una abertura artificial que crea comunicación entre el conducto radicular y los tejidos periodontales, lo que compromete el éxito del tratamiento endodóntico, además de ser uno de los accidentes más desagradables $(5,6)$.

Los localizadores electrónicos apicales (LEA) son considerados las mejores herramientas para determinar la longitud real de los conductos radiculares durante el tratamiento endodóntico $\left({ }^{7}\right)$. Idealmente, los conductos radiculares se preparan y obturan hasta la constricción apical, que es la parte más estrecha con el diámetro más pequeño; es por eso que la precisión de un localizador electrónico apical en la longitud de trabajo resulta de vital importancia $\left.{ }^{8}\right)$. Altunbas et al. $\left({ }^{9}\right)$ refieren que se propuso un método eléctrico para determinar la longitud del conducto; posteriormente, Sunada et al. $\left({ }^{10}\right)$ introdujeron la corriente directa para medir la longitud del conducto, ya que la mucosa oral y el ligamento periodontal tienen la misma resistencia eléctrica para la determinación de la longitud de trabajo.

Con el paso de los años se han desarrollado varias generaciones de LEA. La primera se basó en la resistencia; la segunda, en la impedancia; la tercera utilizó múltiples frecuencias y la cuarta mide la resistencia y la capacitancia por separado, por lo cual utiliza una sola frecuencia a la vez $\left({ }^{3,11,12}\right)$.

Petrucci et al. $\left({ }^{13}\right)$ afirmaron que las soluciones a base de electrolitos contienen iones libres que actúan como conductor eléctrico. Saraswathi et al. $\left({ }^{14}\right)$ observaron que el rendimiento del alginato es superior para la evaluación de los LEA porque sostiene firmemente los dientes y permanece intacto durante la realización del estudio. Topuz et al. $\left({ }^{15}\right)$ refirieron que el alginato tiende a deshidratarse rápidamente, por lo que sugiere realizar las mediciones dentro de los 30 minutos.

El propósito del presente estudio es comparar la precisión de los localizadores electrónicos apicales Propex Pixi y Raypex 6 en la determinación de la longitud de trabajo de piezas dentarias con perforaciones simuladas a diferentes niveles del conducto radicular.

\section{MATERIALES Y MÉTODOS}

Este estudio fue aprobado por el Comité Institucional de Ética en Investigación de la Universidad Científica del Sur (CIEI-CIENTÍFICA) con el código N.o 245-2019-POS8. La muestra estuvo conformada por primeros premolares inferiores unirradiculares que fueron donados por el servicio de odontología del Centro de Salud Salas Guadalupe (Ica).

El diseño fue de tipo experimental in vitro, mientras que, para determinar el tamaño de la muestra, se utilizó la fórmula de comparación de dos medias a un nivel de confianza del $95 \%$, con poder estadístico al $90 \%$, precisión del $2 \%$ y varianza obtenida en la prueba piloto de 1,58 , lo que da como resultado 8 dientes por grupo. Para este estudio, la muestra constó de 36 premolares inferiores (PM) divididos en tres grupos de 12 dientes cada uno. El primer grupo tuvo $\mathrm{PM}$ con dos perforaciones una a nivel medio y otra a nivel apical; el segundo grupo, PM con una perforación a nivel cervical, y el tercer grupo, $\mathrm{PM}$ con una perforación a nivel apical. 


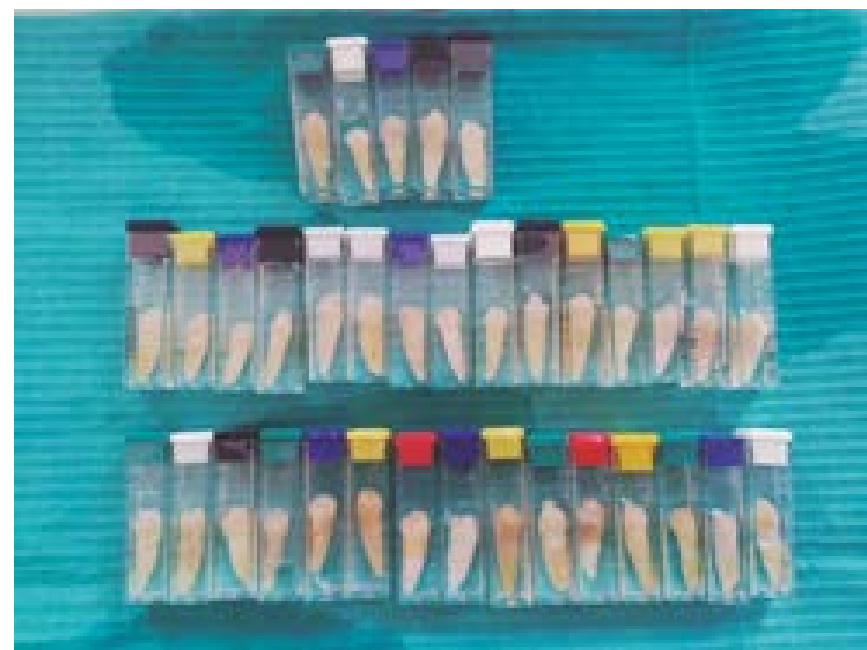

Figura I. Premolares inferiores en frasco con suero fisiológico

Fueron incluidos en el estudio premolares inferiores unirradiculares, de conductos rectos, con ápices cerrados, de un conducto y perforaciones simuladas a diferentes niveles del conducto radicular. Fueron excluidos premolares inferiores con ápices abiertos, reabsorciones, tratamiento endodóntico, hipercementosis, fracturados.

La investigadora (TF) fue capacitada por un especialista. Utilizó 8 dientes para la calibración interobservador y obtuvo un coeficiente de correlación intraclase (CCI) de 0,998 y 0,999 con los LEA Propex Pixi y Raypex 6; para la calibración intraobservador el CCI fue de 0,999 y 0,998 con los LEA Propex Pixi y Raypex 6.

Las piezas dentarias fueron desinfectadas por 2 horas con hipoclorito de sodio al 2,5\%, y luego secadas y almacenadas en frascos con suero fisiológico (figura 1). Además, se enumeraron en la pared vestibular.

Primero,se realizóla apertura cameral;luego,la simulación de las perforaciones desde el exterior de la superficie de la raíz proximal hacia el espacio pulpar en un ángulo de $90^{\circ}$, con una fresa redonda ISO 012, y se comprobó las perforaciones con una radiografía periapical (figura 2). La profundidad de la perforación a nivel cervical y nivel medio fue de $1,5 \mathrm{~mm}$, y la perforación a nivel apical fue de $1 \mathrm{~mm}$ debido a la menor cantidad de tejido dentario en esta zona, según especificaciones de diversos autores $(11-13,16)$.

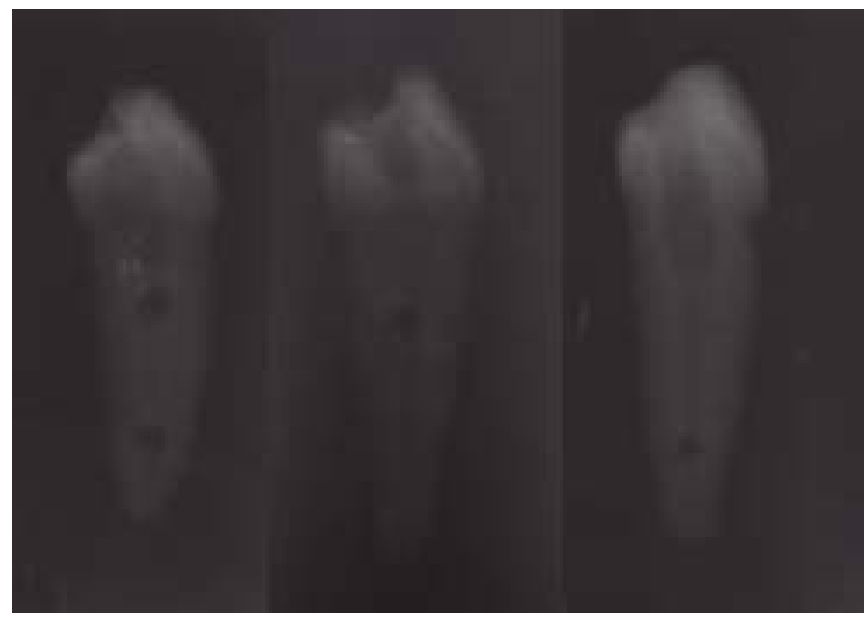

Figura 2. Radiografía periapical de las perforaciones simuladas a diferentes niveles

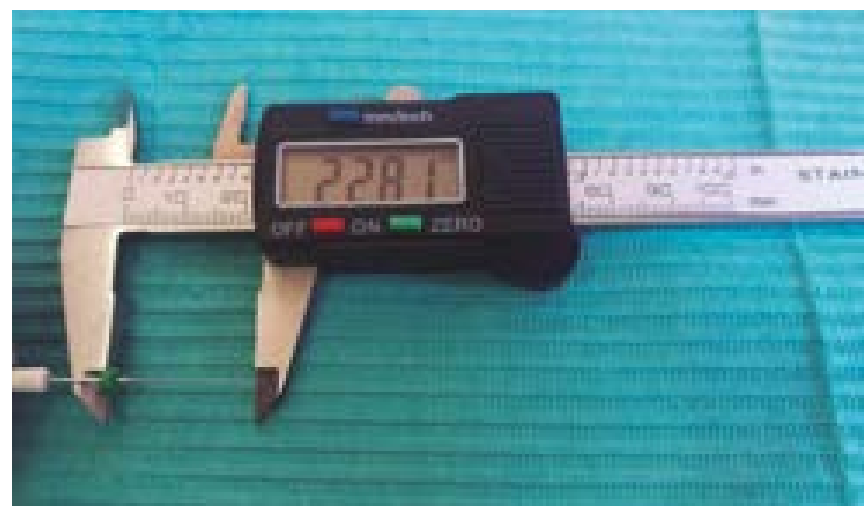

Figura 3. Medición de la lima $n .^{\circ}$ I 5 con el calibrador digital, desde el tope de goma hasta la punta de la lima

Se utilizó una lima n. ${ }^{\circ} 15$ para comprobar el trayecto del conducto radicular (3,11). Posteriormente, la lima fue retirada y, con un calibrador milimétrico digital, se midió la distancia entre la punta de la lima y el tope de goma (figura 3). A esta medida se le disminuyó $1 \mathrm{~mm}$ para obtener la longitud del conducto por visión directa, según lo mencionado por Echevarría y Saraswathi $\left({ }^{12,14}\right)$.

Se utilizó alginato para simular la cavidad oral $\left({ }^{10,11}\right.$, $\left.{ }^{17}\right)$ y este fue depositado en un recipiente de plástico donde se colocaron 6 dientes dejando $5 \mathrm{~mm}$ libres de la corona con un tiempo de trabajo de 30 minutos, según la recomendación de Topuz et al. $\left.{ }^{15}\right)$. Se colocó el gancho labial y se humedeció el conducto con 0,2 $\mathrm{ml}$ de hipoclorito de sodio al 2,5\% (4,6). Finalmente, se colocó el clip sobre la lima, a fin de cerrar el circuito y determinar la medición $(6,11,12)$. 


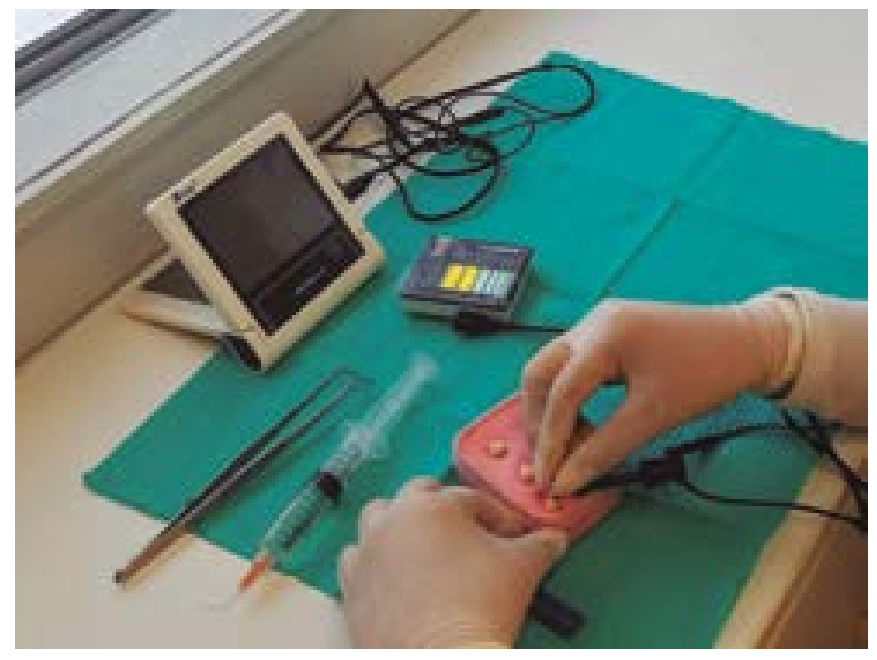

Figura. 4. Medición del diente con el localizador electrónico apical Propex Pixi

Según indicación del fabricante, al observar $\operatorname{APEX}(0,0)$ en el localizador electrónico apical Propex Pixi, se le restan 0,5 milímetros a la longitud obtenida (figura 4). En el localizador electrónico apical Raypex 6, cuando se observan tres barras verdes marcadas en la pantalla esto significa que la lima ha llegado a la posición cercana al foramen apical (figura 5) y se mide la longitud obtenida. Posteriormente, se registraron los valores obtenidos en una ficha recolección de datos para su evaluación.

Varios autores indican que las mediciones de los LEA se consideraron "precisas" cuando fueron de 0,50 mm; "aceptables", entre 0,51 mm y $1 \mathrm{~mm}, \mathrm{y}$ "equivocadas" si fueron mayores a $1 \mathrm{~mm}\left({ }^{11,12,14}\right)$.

Mediante el uso del programa IBM SPSS Statistics 25, se realizó la estadística descriptiva que consistió en obtener la media, la desviación estándar y el rango de cada uno de los tres grupos. Posteriormente, para la estadística inferencial, se utilizaron la prueba no paramétrica $U$ de Mann-Whitney y el análisis de varianza de Friedman para muestras relacionadas.

\section{RESULTADOS}

Con respecto a la descripción de la longitud (mm) de las piezas dentarias obtenidas con los LEA y el tipo de perforación simulada en los diferentes niveles del conducto radicular, se obtuvo que el mayor valor

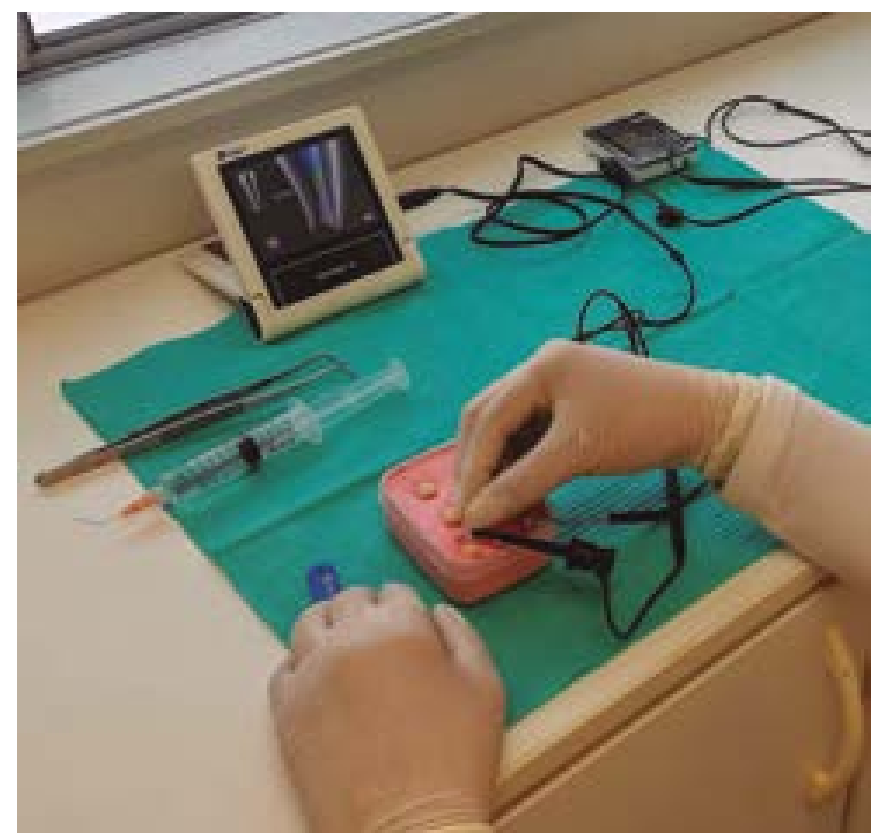

Figura 5. Medición del diente con el localizador electrónico apical Raypex 6

encontrado de la media se halló en el grupo de LEA Raypex 6, con 21,74 mm, en dientes con una perforación a nivel cervical, y el menor valor se halló en el grupo de LEA Propex Pixi, con 20,16 mm, en dientes con dos perforaciones, una a nivel medio y la otra a nivel apical (tabla 1).

En la comparación de la longitud real del conducto con respecto a la precisión de la longitud ( $\mathrm{mm}$ ) de las piezas dentarias con dos perforaciones con los LEA Propex Pixi y Raypex 6, se encontraron diferencias estadísticamente significativas con un $\mathrm{p}<0,05$ (tabla 2).

Con respecto a la precisión de las longitudes (mm) de las piezas dentarias en las diferentes perforaciones simuladas del conducto radicular, se observó que el localizador electrónico apical (LEA) Propex Pixi sobrestima la longitud en menos de medio milímetro en todos los casos y el LEA Raypex 6 subestima la longitud en menos de medio milímetro (tabla 3 ).

En cuanto a la comparación de la precisión de los valores de la media de las longitudes $(\mathrm{mm})$ de las piezas dentarias obtenidas con los LEA Propex Pixi y Raypex 6 en perforaciones simuladas a diferentes niveles del conducto radicular, se utilizó la prueba no paramétrica 
Tabla 1. Descripción de la longitud ( $\mathrm{mm}$ ) de las piezas dentarias obtenidas con los localizadores Propex Pixi y Raypex 6 en perforaciones simuladas a diferentes niveles del conducto radicular

\begin{tabular}{|c|c|c|c|c|c|}
\hline $\begin{array}{c}\text { Localizador electrónico apical y } \\
\text { nivel de perforación }\end{array}$ & $\mathbf{n}$ & Media & D. E. & Mínimo & Máximo \\
\hline $\begin{array}{l}\text { Propex Pixi perforación a nivel medio } \\
\text { y nivel apical }\end{array}$ & 12 & 20,16 & 1,26 & 18,42 & 3,16 \\
\hline $\begin{array}{l}\text { Raypex } 6 \text { perforación a nivel medio y } \\
\text { nivel apical }\end{array}$ & 12 & 20,69 & 1,39 & 18,48 & 23,84 \\
\hline Propex Pixi perforación a nivel cervical & 12 & 21,45 & 1,62 & 18,98 & 23,78 \\
\hline Raypex 6 perforación a nivel cervical & 12 & 21,74 & 1,57 & 19,09 & 23,98 \\
\hline Propex Pixi perforación a nivel apical & 12 & 21,21 & 1,47 & 19,38 & 23,75 \\
\hline Raypex 6 perforación a nivel apical & 12 & 21,64 & ।,7। & 19,43 & 24,55 \\
\hline
\end{tabular}

U de Mann-Whitney, mediante la cual se encontraron diferencias estadísticamente significativas con un $\mathrm{p}<$ 0,05 (tabla 4).

\section{DISCUSIÓN}

El presente estudio empleó la radiografía periapical como herramienta de apoyo para corroborar la precisión de los LEA en la determinación de la longitud de trabajo. Respecto de la precisión de las longitudes de trabajo, el localizador Propex Pixi sobrestima la longitud y el Raypex 6 la subestima, en ambos casos en menos de medio milímetro. Cabe resaltar que la precisión se determinó por la diferencia encontrada entre la medida real del conducto radicular y la medida obtenida con el localizador electrónico apical (LEA).

El localizador electrónico apical Propex Pixi (Dentsply Maillefer) y el localizador electrónico apical Raypex 6 (VDW) presentaron diferencias significativas. Estos resultados difieren de los encontrados por Khatri et al. $(3)$, quienes demostraron que no existe diferencia significativa en perforaciones radiculares entre el localizador Gold VDW y el Localizador Raypex 6. Probablemente, la discrepancia de resultados se deba a que las perforaciones fueron realizadas en el lado distal de la raíz mesial de molares inferiores, porque es considerada zona de peligro de las piezas dentarias.

En el estudio realizado por Srivastava et al. $\left({ }^{5}\right)$ se menciona que el Raypex6 Apex locater detectó las perforaciones de
Tabla 2. Comparación de las longitudes ( $\mathrm{mm}$ ) obtenidas con los localizadores Propex Pixi y Raypex 6 en perforaciones simuladas a diferentes niveles del conducto radicular con la longitud real del conducto

\begin{tabular}{llc}
\hline Localizador electrónico apical y tipo de perforación & Media & p \\
\hline Longitud real del conducto & 20,48 & \\
\hline Propex Pixi perforación nivel medio y apical & 20,16 & $<0,001$ \\
\hline Raypex 6 perforación nivel medio y apical & 20,69 & \\
\hline Longitud real del conducto & 21,52 & \\
\hline Propex Pixi perforación nivel cervical & 21,45 & 0,001 \\
\hline Raypex 6 perforación nivel cervical & 21,74 & \\
Longitud real del conducto & 21,43 & \\
\hline Propex Pixi perforación nivel apical & 21,21 & 0,005 \\
\hline Raypex 6 perforación nivel apical & 21,64 & \\
\hline
\end{tabular}

Prueba Friedman

Tabla 3. Descripción de la precisión de las longitudes ( $\mathrm{mm}$ ) de las piezas dentarias obtenidas con los localizadores Propex Pixi y Raypex 6 en perforaciones simuladas a diferentes niveles del conducto radicular

\begin{tabular}{lcccccc}
$\begin{array}{l}\text { Localizador electrónico apical y } \\
\text { nivel de perforación }\end{array}$ & $\mathbf{n}$ & Media & D. E. & Mínimo & Máximo \\
\hline $\begin{array}{l}\text { Precisión Propex Pixi perforación } \\
\text { nivel medio y nivel apical }\end{array}$ & 12 & 0,31 & 0,29 & 0,00 & 0,80 \\
$\begin{array}{l}\text { Precisión Raypex 6 perforación } \\
\text { nivel medio y nivel apical }\end{array}$ & 12 & $-0,21$ & 0,32 & $-0,84$ & 0,39 \\
$\begin{array}{l}\text { Precisión Propex Pixi perforación } \\
\text { nivel cervical }\end{array}$ & 12 & 0,08 & 0,14 & $-0,12$ & 0,36 \\
$\begin{array}{l}\text { Precisión Raypex 6 perforación } \\
\text { nivel cervical }\end{array}$ & 12 & $-0,22$ & 0,32 & $-0,84$ & 0,19 \\
$\begin{array}{l}\text { Precisión Propex Pixi perforación } \\
\text { nivel apical }\end{array}$ & 12 & 0,22 & 0,28 & 0,00 & 0,80 \\
$\begin{array}{l}\text { Precisión Raypex 6 perforación } \\
\text { nivel apical }\end{array}$ & 12 & $-0,21$ & 0,47 & $-0,98$ & 0,76 \\
\hline
\end{tabular}

Tabla 4. Comparación de la precisión de las longitudes $(\mathrm{mm})$ de las piezas dentarias obtenidas con los localizadores Propex Pixi y Raypex 6 en perforaciones simuladas a diferentes niveles del conducto radicular

\begin{tabular}{lcc}
\multicolumn{1}{c}{ Precisión } & Media & P \\
Propex Pixi perforación nivel medio y nivel apical & 0,31 & \\
Raypex 6 perforación nivel medio y nivel apical & $-0,21$ & $<0,001$ \\
Propex Pixi perforación nivel cervical & 0,08 & \multirow{2}{*}{0,008} \\
Raypex 6 perforación nivel cervical & $-0,22$ & \\
Propex Pixi perforación nivel apical & 0,22 & \multirow{2}{*}{0,006} \\
Raypex 6 perforación nivel apical & $-0,21$ & \\
\hline
\end{tabular}

Prueba $\cup$ de Mann-Whitney 
la raíz de manera más significativa y puede ser mejor que Propex Pixi, a diferencia del presente estudio que muestra que el localizador electrónico apical Propex Pixi tuvo mejor precisión para determinar la longitud de trabajo en piezas dentarias perforadas artificialmente a diferentes niveles del conducto radicular. La similitud que presenta se consigue porque hay una coincidencia en el nivel de la perforación de ambos estudios.

Se obtuvieron valores negativos con medidas de precisión menores a medio milímetro utilizando el LEA Raypex 6 con una media de $-0.21 \mathrm{~mm}$, resultado parecido al del estudio de Gürel et al. $\left({ }^{18}\right)$, quienes determinaron que Raypex 6 tuvo una media de 0,37 mm. La semejanza podría deberse al uso de la misma herramienta de medición y que, al ser de cuarta generación, logra una determinación eficaz de la longitud de trabajo.

E1 LEA Raypex 6 subestima la precisión de la longitud de trabajo en menos de medio milímetro y obtiene valores negativos dentro del rango de precisión $-0,21$ a $-0,22$ $\mathrm{mm}$. El resultado encontrado por Khandewal $\left({ }^{19}\right)$ señala que la subestimación de la determinación de la longitud de trabajo con el LEA Raypex 5 fue de $0,72 \mathrm{~mm}$. No se encontraron estudios que demuestren el porqué de la subestimación en la longitud de trabajo.

En este estudio se encontró que el localizador electrónico apical Propex Pixi (Dentsply Maillefer) sobreestima la precisión de la longitud de trabajo en menos de medio milímetro, con lo que obtiene valores positivos en la medición, semejantes a los del estudio de Somma et al. $\left({ }^{20}\right)$, el cual menciona que los localizadores Raypex y ProPex dieron valores positivos y negativos utilizando una precisión de $\pm 0,5 \mathrm{~mm}$ de la longitud real del conducto. Los resultados obtenidos son similares porque utilizan LEA iguales para determinar la longitud de trabajo en dientes que presentan perforaciones.
Se recomienda realizar otros estudios con dientes perforados y el uso de irrigantes para conocer si influyen en la medición de la longitud de trabajo, así como otro tipo de LEA y comparar los resultados. También se pueden llevar a cabo estudios utilizando sangre de carnero, porque es similar a la del ser humano y permitirá determinar la efectividad de los LEA $\left(^{(21}\right)$.

Estos resultados permitirán orientar al clínico a la elección más conveniente de un localizador electrónico apical en dientes que presenten perforaciones para obtener una determinación favorable de la longitud de trabajo. Cabe mencionar que, desde el punto de vista social, la selección de un buen localizador electrónico apical en estos casos puede ser muy beneficioso para los pacientes, ya que el tiempo de atención podría disminuir y serían atendidos eficazmente por el profesional.

\section{CONCLUSIÓN}

En el estudio, se encontraron diferencias significativas entre el localizador electrónico apical Propex Pixi y el Raypex 6, que sobreestimaron y subestimaron, respectivamente, la longitud del conducto en menos de medio milímetro.

Contribución de autoría: Teresa Flora Astocaza Reátegui, Lourdes Rocío Ayarza Flórez y Carmen Rosa García Rupaya han participado en la concepción, recolección de datos, redacción y aprobación de la versión final del artículo.

Fuente de financiamiento: Autofinanciado.

Conflictos de intereses: Las autoras declaran no tener conflictos de intereses. 


\section{REFERENCIAS BIBLIOGRÁFICAS}

1. Cruz ATG, Wichnieski C, Carneiro E, da Silva Neto UX, Gambarini G, Piasecki L. Accuracy of 2 endodontic rotary motors with integrated apex locator. J Endod. 2017 Oct; 43 (10): 1716-9. doi: 10.1016/j.joen.2017.05.012

2. Zmener O, Luberti R. Evaluación radiográfica de 80 casos de perforación radicular. Endodoncia. 2009 Abr-Jun; 27 (2): 68-74. doi: http://www.medlinedental.com/pdf-doc/ENDO/v27-2-4. pdf

3. Khatri MP, Ghivari SB, Pujar M, Faras R, Gopeshetti P, Vanti A. Accuracy of two electronic apex locators in locating root perforations in curved canals in dry and wet conditions: A comparative in vitro study. Dent Res J (Isfahan). 2019 Nov 12; 16 (6): 407-12. doi: https://pubmed.ncbi.nlm.nih.gov/31803387/

4. Vera A. Estudio comparativo de dos localizadores apicales para la determinación de las perforaciones radiculares in vitro [Tesis]. Quito: Universidad Central del Ecuador; 2017. doi: http://www. dspace.uce.edu.ec/bitstream/25000/9115/1/T-UCE-0015-512. pdf

5. Srivastava V, Jain N, Bagchi S, Pal Singh M. Evaluation of the use of sixth generation apex locators as a diagnostic tool to detect root perforations. IJDMS. 2015 Oct-Dec; 2 (4): 10. doi: 10.5958/2394-4196.2015.00025.4

6. CruzA.Tratamientodelasperforacionesradiculares[Tesis].Sevilla: Universidad de Sevilla; 2017/2018. doi:https://idus.us.es/bitstream/ handle/11441/78169/TFG\%20ALBERTO\%20CRUZ\%20 VALLE.pdf?sequence=1\&isAllowed $=y \#: ~:$ text $=$ un $\% 20$ buen $\% 20$ pron $\%$ C3\%B3stico.-,1.3.TRATAMIENTO,trav\%C3\%A9s\%20 de\%20los\%20tejidos\%20perirradiculares

7. De Vasconcelos BC, Veríssimo Chaves RD, Vivacqua-Gomes N, Candeiro GT, Bernardes RA, Vivan RR, Duarte MA. Ex vivo evaluation of the accuracy of electronic foramen locators in root canals with an obstructed apical foramen. J Endod. 2015 Sep; 41 (9): 1551-4. doi: 10.1016/j.joen.2015.06.009

8. Cimilli H, Aydemir S, Arican B, Mumcu G, Chandler N, Kartal N. Accuracy of the Dentaport ZX apex locator for working length determination when retreating molar root canals. Aust Endod J. 2014 Apr; 40 (1): 2-5. doi: 10.1111/aej.12010

9. Altunbas D, Kustarci A, Arslan D, Er K. In vitro comparison of four different electronic apex locators to determine the major foramen using the clearing technique. Niger J Clin Pract. 2014 Nov-Dec; 17 (6): 706-10. doi: 10.4103/1119-3077.144382

10. Sunada I. New method for measuring the length of the root canal. J Dent Res. 1962 March-April; 41 (2): 375-87. doi: 10.1177 / 00220345620410020801

11. Toledo E. Estudio in vitro de la eficacia de dos localizadores electrónicos de ápice:YS-RZ300 y RPEX 6 en dientes premolares unirradiculares [Tesis]. Lima: UAP; 2018. doi: https://docplayer. es/138273396-Tesis-estudio-in-vitro-de-la-eficacia-de-doslocalizadores-electronicos-de-apice-ys-rz300-y-rpex-6-endientes-premolares-unirradiculares.html
12. Echevarría I. Eficacia in vitro de dos localizadores foraminales: Easy apex y Miniapex en la localización de la unión cemento-dentina (U.C.D) en premolares inferiores unirradiculares [Tesis]. Arequipa: UCSM; 2016. doi: http://tesis.ucsm.edu.pe/repositorio/bitstream/handle/ UCSM/5489/64.2640.O.pdf?sequence=1\&isAllowed=y

13. Petrucci R, Herring F, Madura J, Bissonnette C. Química general. Principios y aplicaciones modernas. 10.a ed. Madrid: Pearson; 2011. doi: https://quimica247403824.files.wordpress. com/2018/11/quimica_general_petrucci.pdf

14. Saraswathi V, Kedia A, Purayil TP, Ballal V, Saini A. Comparative evaluation of the accuracy of two electronic apex locators in determining the working length in teeth with simulated apical root resorption: An in vitro study. J Conserv Dent. 2016 SepOct; 19 (5): 402-5. doi: 10.4103/0972-0707.190002

15. Topuz O, Uzun O, Tinaz AC, Sadik B. Accuracy of the apex locating function of TCM Endo $\mathrm{V}$ in simulated conditions: a comparison study. Oral Surg Oral Med Oral Pathol Oral Radiol Endod. 2007 Mar; 103 (3): e73-6. doi: 10.1016/j. tripleo.2006.09.004

16. Altunbaş D, Kuştarcı A, Toyoğlu M. The influence of various irrigants on the accuracy of 2 electronic apex locators in locating simulated root perforations. J Endod. 2017 Mar; 43 (3): 439-42. doi: 10.1016/j.joen.2016.10.031

17. Taneja S, Kumar M, Soi S, Gogia H. Comparative evaluation of accuracy of three electronic apex locators in different simulated clinical conditions- an invitro study. An of Med and Health Sci Res. 2017 May-June; 7: 190-4. doi: https:/www. amhsr.org/articles/comparative-evaluation-of-accuracy-ofthree-electronic-apex-locators-in-different-simulated-clinicalconditions-an-invit.pdf

18. Gurel MA, Helvacioglu Kivanc B, Ekici A. A comparative assessment of the accuracies of Raypex 5, Raypex 6, iPex and iPex II electronic apex locators: An in vitro study. J Istanb Univ Fac Dent. 2017 Jan 2; 51 (1): 28-33. doi: 10.17096/jiufd.61309

19. Khandewal D, Ballal NV, Saraswathi MV. Comparative evaluation of accuracy of 2 electronic Apex locators with conventional radiography: an ex vivo study. J Endod. 2015 Feb; 41 (2): 201-4. doi: 10.1016/j.joen.2014.10.011

20. Somma F, Castagnola R, Lajolo C, Paternò Holtzman L, Marigo L. In vivo accuracy of three electronic root canal length measurement devices: Dentaport ZX, Raypex 5 and ProPex II. Int Endod J. 2012 Jun; 45 (6): 552-6. doi: 10.1111/j.13652591.2011.02010.x

21. Russell FM, Biribo SS, Selvaraj G, Oppedisano F, Warren S, Seduadua A, Mulholland EK, Carapetis JR. As a bacterial culture medium, citrated sheep blood agar is a practical alternative to citrated human blood agar in laboratories of developing countries. J Clin Microbiol. 2006 Sep; 44 (9): 3346-51. doi: 10.1128/JCM.02631-05 